\title{
Phase-resolved spectroscopic study of the isolated neutron star RBS 1223 (1RXS J130848.6+212708)^
}

\author{
V. Hambaryan ${ }^{1}$, V. Suleimanov ${ }^{2,3}$, A. D. Schwope ${ }^{4}$, R. Neuhäuser ${ }^{1}$, K. Werner ${ }^{2}$, and A. Y. Potekhin ${ }^{5,6,7}$ \\ 1 Astrophysikalisches Institut und Universitäts-Sternwarte, Universität Jena, Schillergäßchen 2-3, 07745 Jena, Germany \\ e-mail: vvh@astro.uni-jena.de \\ ${ }^{2}$ Institute for Astronomy and Astrophysics, Kepler Center for Astro and Particle Physics, Eberhard Karls University, Sand 1, \\ 72076 Tübingen, Germany \\ 3 Kazan Federal University, Kremlevskaja Str., 18, Kazan 420008, Russia \\ ${ }^{4}$ Leibniz-Institut für Astrophysik Potsdam, An der Sternwarte 16, 14482 Potsdam, Germany \\ 5 Ioffe Physical-Technical Institute, Politekhnicheskaya Str., 26, St. Petersburg 194021, Russia \\ 6 CRAL, École Normale Supérieure de Lyon, UMR CNRS No. 5574, Université de Lyon, 69364 Lyon Cedex 07, France \\ 7 Isaac Newton Institute of Chile, St. Petersburg Branch, Russia
}

Received 23 June 2011 / Accepted 15 August 2011

\begin{abstract}
Aims. We constrain the mass-to-radius ratio of isolated neutron stars by performing a spin-phase resolved X-ray spectroscopic analysis.

Methods. We combined the data from all observations of RBS 1223 (1RXS J130848.6+212708) conducted by XMM-Newton EPIC pn with the same instrumental setup in 2003-2007 to form spin-phase resolved spectra. We implemented a number of complex models of neutron stars with strongly magnetized $\left(B_{\text {pole }} \sim 10^{13}-10^{14} \mathrm{G}\right)$ surfaces, various temperature and magnetic-field distributions around their magnetic poles, and a partially ionized hydrogen-thin atmosphere above into the X-ray spectral fitting package XSPEC for simultaneous fitting of phase-resolved spectra. A Markov-chain Monte Carlo (MCMC) approach is also applied to verify the results of fitting and estimating of parameters in multi-parameter models.

Results. The spectra of different rotational phase intervals and light curves of different energy bands with high signal-to-noise ratio show a high complexity. The spectra can be parameterized with a Gaussian absorption-line superimposed on a blackbody spectrum, while the pulsed fraction of light curves with double-humped shape strongly depend upon the energy band (13-42\%), which indicates that radiation emerges from at least two emitting areas.

Conclusions. A model with a condensed iron surface and partially ionized hydrogen-thin atmosphere above allows us to fit simultaneously the observed general spectral shape and the broad absorption feature observed at $0.3 \mathrm{keV}$ in different spin phases of RBS 1223 . We constrain some physical properties of the X-ray emitting areas, i.e. the temperatures $\left(T_{\mathrm{p} 1} \sim 105 \mathrm{eV}, T_{\mathrm{p} 2} \sim 99 \mathrm{eV}\right)$, magnetic field strengths $\left(B_{\mathrm{p} 1} \approx B_{\mathrm{p} 2} \sim 8.6 \times 10^{13} \mathrm{G}\right)$ at the poles, and their distribution parameters $\left(a_{1} \sim 0.61, a_{2} \sim 0.29\right.$, indicating an absence of strong toroidal magnetic field component). In addition, we are able to place some constraints on the geometry of the emerging X-ray emission and the gravitational redshift $\left(z=0.16_{-0.01}^{+0.03}\right)$ of RBS 1223.
\end{abstract}

Key words. stars: neutron - methods: data analysis - X-rays: stars - stars: atmospheres - stars: individual: RBS 1223

\section{Introduction}

The study of thermally emitting, radio-quiet, nearby, isolated neutron stars (INSs) may have an important impact on our understanding of the physics of neutron stars. Observations and modeling of thermal emission from INSs can provide information not only on the physical properties, such as the magnetic field, temperature, and chemical composition of the regions where this radiation is produced, but also information on the properties of matter at higher densities deeper inside the star.

In particular, measuring the gravitational redshift of an identified spectral feature in the spectrum of thermal radiation emitted from the INS surface/atmosphere may provide a useful constraint of theoretical models of the equations of state for superdense matter. Independent of the estimate of the INS radius (e.g. Trümper et al. 2004) from the thermal spectrum of an INS,

* Based on observations obtained with XMM-Newton, an ESA science mission with instruments and contributions directly funded by ESA Member States and NASA. measurement of the redshift may allow us to directly estimate the mass-to-radius ratio.

Much more information may be extracted by studying spinphase-resolved spectra of high signal-to-noise ratio $(\mathrm{S} / \mathrm{N})$ and fitting them with model spectra of radiation emitted from highly magnetized INS surface layers.

The soft X-ray source RBS 1223 (1RXS J130848.6+ 212708) was originally discovered during the ROSAT All-Sky Survey by Schwope et al. (1999). It shared common characteristics with other members of the small group (so far seven discovered by ROSAT) of thermally emitting and radio-quiet, nearby INSs, traditionally dubbed XDINS (meaning "X-ray dim INS") or Magnificent Seven (see, e.g., reviews by Haberl 2007; Mereghetti 2008; Turolla 2009, and references therein): soft spectra, well-described by blackbody radiation with temperatures 60-120 eV, no other spectral features, no association with $\mathrm{SNR}$, no radio emission, no $\mathrm{X}$-ray pulsations, and a very high $\mathrm{X}$-ray to optical flux ratio. That these sources are old INS reheated by accretion from either the interstellar medium or young 
cooling stars appears possible. Intensive X-ray observations with mainly XMM-Newton in addition to Chandra, as well as optical/UV observations of the most probable counterparts, have revised this picture in parts and provided interesting physical insight.

In six of these objects, X-ray pulsations have been found with periods clustered at 3-11 s, and period derivatives have been measured for five of them. In a classic $P-\dot{P}$ diagram, the XDINSs are found in-between radio pulsars and magnetars (e.g., Mereghetti 2008). The inferred magnetic field strengths are above $10^{13} \mathrm{G}$.

Imaging CCD spectroscopy with XMM-Newton has uncovered absorption features in at least three, probably in six stars. At the present energy resolution $(F W H M \sim 50-150 \mathrm{eV}$ in the range $0.2-2.0 \mathrm{keV}$ ), they can be formally well fitted as Gaussian absorption lines, which are usually related to ion cyclotron lines. Their interpretation is not unique, and alternatives such as magnetically shifted atomic transitions or electron cyclotron resonances have also been debated. For some models, the inferred magnetic field strengths are again above $10^{13} \mathrm{G}$ (Haberl 2007).

Among them, RBS 1223 is a special case, some sort of outlier, that is qualitatively different from the typical pattern observed in the sample of XDINSs. The rotational phase-folded light curve has a double-humped shape and the largest pulsed fraction $^{1}$ ( $\sim 19 \%$ in the $0.2-1.2 \mathrm{keV}$ energy range). Moreover, the separation of maxima by less than 180 degrees, significantly different count-rates of minima, and the variation in the blackbody apparent temperatures between 80 and $90 \mathrm{eV}$ over the spin cycle are evidence for, at least, two emitting areas with some temperature distribution over the neutron star surface (Schwope et al. 2005, 2007).

We note that the above-mentioned observed absorption feature at $\sim 0.3 \mathrm{keV}$ in the spectrum of RBS 1223 has the largest equivalent width $(\sim 0.2 \mathrm{keV}$, Schwope et al. 2007) among all XDINSs. Moreover, the spectral analysis of the average XMM-Newton spectrum of RBS 1223 (Schwope et al. 2007) based on the two first observations (see Table 1) showed the possible presence of a second feature in the X-ray spectrum. Its existence, however, is not uniquely proven because of some remaining calibration uncertainties and the poorly defined continuum at high energies because of the lack of photons (lower $\mathrm{S} / \mathrm{N}$ ).

New observational sets (see Table 1) have become publicly available and a number of new detailed models of emergent spectra of highly magnetized INSs have been developed and become available (Ho et al. 2009; Suleimanov et al. 2010a).

Preliminary analysis of the phase-averaged spectrum of RBS 1223 was performed by Pérez-Azorín et al. (2006b). They mentioned that there is a possibility of good fits with quadrupolar magnetic fields, although not excluding a condensed surface

\footnotetext{
1 The pulsed fraction, used in this paper, is defined as

$\mathrm{PF} \equiv \frac{\mathrm{CR}_{\max }-\mathrm{CR}_{\min }}{\mathrm{CR}_{\max }+\mathrm{CR}_{\min }}$,

where CR is the count rate. Note that this definition of a pulsed fraction may be misleading in the case of complex shaped light curves, a peak/minimum flux may appear at different phases in different energy ranges. The following quantity, i.e. semi-amplitude of modulation, might instead be an appropriate descriptor of the pulsed emission:
}

$A \equiv \frac{\sum_{i}\left|\mathrm{CR}_{i}-\left\langle\mathrm{CR}_{i}\right\rangle\right|}{\sum_{i} \mathrm{CR}_{i}}$,

where $\mathrm{CR}_{i}$ is a count rate per phase bin.

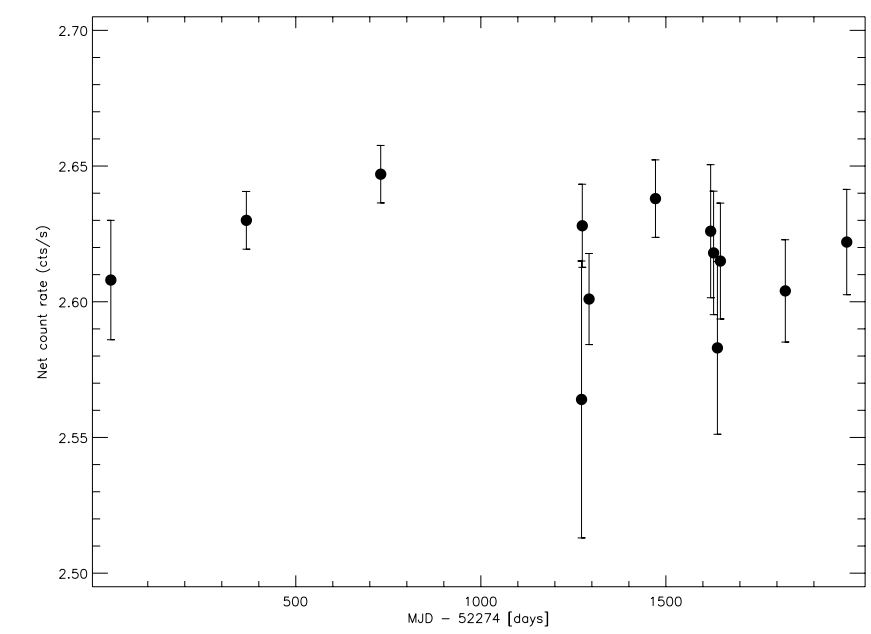

Fig. 1. Observed count rates of RBS 1223 in the energy band 0.16-2.0 keV in different XMM-Newton EPIC pn observations.

model with a hydrogen atmosphere, including vacuum polarization effects (van Adelsberg \& Lai 2006).

To explain unusual observed properties of RBS 1223, Suleimanov et al. (2010a, hereafter Paper I) studied various local models of the emitting surface of this INS. They considered three types of models: naked condensed surfaces, semi-infinite partially ionized hydrogen atmospheres with vacuum polarization, and that took into account partial mode conversion (see details in Suleimanov et al. 2009), in addition to thin atmospheres above the condensed iron surface. They also created a code for modeling integral phase-resolved spectra and light curves of rotating neutron stars. This code takes into account general relativistic effects and allows one to consider various temperature and magnetic field distributions. Analytical approximations of the three considered types of local spectra were used for this modeling. It was qualitatively shown that only a thin model atmosphere above a condensed iron surface can explain the observed equivalent width of the absorption feature and the pulsed fraction.

In this paper, we use the code developed in Paper I to perform a comprehensive study of co-added high-S/N and phaseresolved XMM-Newton EPIC pn spectrum of RBS 1223, despite some possible small variations in brightness shown by this INS (see Fig. 1).

\section{Observations and data reduction}

The source RBS 1223 has been observed many times by XMM-Newton (Table 1). Here we focus on the data collected with EPIC pn (den Herder et al. 2001) from the 12 publicly available observations, with the same instrumental setup (full frame mode, thin 1 filter, positioned on axis), in total presenting about $175 \mathrm{ks}$ of effective exposure time.

The data were reduced using standard threads from the $X M M$-Newton data analysis package SAS version 10.0.0. We reprocessed all publicly available data (see Table 1) with the standard metatask epchain. To determine good time intervals free of background flares, we applied a filtering expression to the background light curves, performing visual inspection. This reduced the total exposure time by $\sim 30 \%$. Solar barycenter-corrected source and background photon event files and spectra were produced from the cleaned SINGLE ${ }^{2}$ events, using an extraction radius of $30^{\prime \prime}$ in all pointed observations. We also extracted

\footnotetext{
${ }^{2}$ See XMM-Newton Users Handbook.
} 
Table 1. XMM-Newton EPIC pn observations of RBS 1223.

\begin{tabular}{lccc}
\hline \hline Obs. ID & $\begin{array}{c}\text { Obs. date } \\
\text { MJD }\end{array}$ & $\begin{array}{c}\text { Exposure } \\
\text { ks }\end{array}$ & $\begin{array}{c}\text { Effective exposure } \\
\text { ks }\end{array}$ \\
\hline 0157360101 & 52640.4271023 & 28.910 & 25.966 \\
0163560101 & 53003.4674787 & 32.114 & 26.436 \\
0305900201 & 53546.0889925 & 16.806 & 11.443 \\
0305900301 & 53548.0919318 & 14.812 & 12.682 \\
0305900401 & 53566.4161765 & 14.814 & 10.574 \\
0305900601 & 53745.8803029 & 16.845 & 14.702 \\
0402850301 & 53894.9783016 & 7.419 & 4.761 \\
0402850401 & 53902.9489095 & 8.421 & 5.558 \\
0402850501 & 53913.2292603 & 12.517 & 2.801 \\
0402850701 & 53921.2857528 & 10.411 & 6.366 \\
0402850901 & 54096.6780925 & 9.315 & 8.011 \\
0402851001 & 54262.6501350 & 10.920 & 8.462 \\
\hline
\end{tabular}

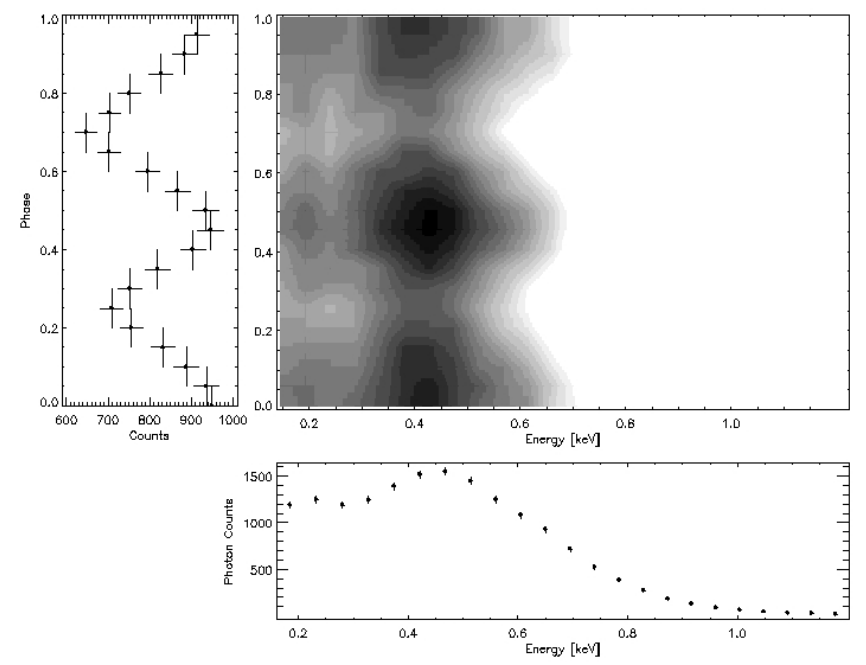

Fig. 2. Energy-phase image of RBS 1223 combined from different observations. Rotational phase-folded light curve in the broad energy, 0.2-2.0 keV, band (left panel) and the phase-averaged spectrum (bottom panel) are shown.

light curves of RBS 1223 and corresponding backgrounds from nearby, source-free regions. We then used the SAS task epiclccorr to correct observed count rates for various sorts of detector inefficiencies (vignetting, bad pixels, dead time, effective areas, etc.) in different energy bands for each pointed observation (see Fig. 1).

For each registered photon, the corresponding rotational phase was computed according to the phase-coherent timing solution provided by Kaplan \& van Kerkwijk (2005). We produced spin phase-folded light curves in different energy bands and spectra corresponding to different phase intervals for each pointed observation. Finally, the latter ones were co-added for the same intervals of phases to create the combined phaseresolved spectra of RBS 1223 (Fig. 2).

We note that spectral responses and effective areas of these 12 different observations were almost indistinguishable.

\section{Data analysis}

First, we have fitted the phase-averaged, high S/N spectral data, collected from different observations (Fig. 3 and Table 2), using a combination of an absorbed blackbody and a Gaussian absorption-line multiplicative component model (in XSPEC phabs*bbodyrad*gabs).
Table 2. Fitting results for model phabs*gabs*bbodyrad $(\tau=0.612 \pm$ $\left.0.08, \sigma=0.155 \pm 0.008, N_{\mathrm{H}}=(1.8 \pm 0.0035) \times 10^{18} \mathrm{~cm}^{-2}\right)$.

\begin{tabular}{lccc}
\hline \hline Phase interval & $\begin{array}{c}\mathrm{kT} \\
(\mathrm{keV})\end{array}$ & $\begin{array}{c}\text { Line center } \\
(\mathrm{keV})\end{array}$ & Remarks \\
\hline $0.20-0.35$ & $0.084 \pm 0.001$ & $0.25 \pm 0.01$ & First minimum \\
$0.35-0.65$ & $0.088 \pm 0.001$ & $0.26 \pm 0.01$ & Secondary peak \\
$0.65-0.80$ & $0.083 \pm 0.001$ & $0.24 \pm 0.01$ & Second minimum \\
$0.0-0.2,0.8-1.0$ & $0.088 \pm 0.001$ & $0.29 \pm 0.01$ & Primary peak \\
$0.00-1.0$ & $0.086 \pm 0.001$ & $0.27 \pm 0.01$ & Phase averaged \\
\hline
\end{tabular}

In the spectral energy range of $0.16-2.0 \mathrm{keV}$, we obtained a statistically acceptable fit with the reduced $\chi^{2}=1.1$.

We also performed a fit with the above-mentioned spectral model for four phase intervals, including maxima and minima of the double-humped phase-folded light curve, simultaneously. The fitted parameters are presented in Table 2, which illustrates the dependence of both the blackbody apparent temperature and the Gaussian absorption feature center on rotational phase. Next, we divided the broad energy band into four energy regions $(0.16-0.5,0.5-0.6,0.6-0.7$, and $0.7-2.0 \mathrm{keV})$ and constructed rotational phase-folded light curves (Fig. 4). It is also noteworthy that the higher the considered spectral energy range, the larger the pulsed fraction. These spin phase-folded light curves may serve as rough estimates of the viewing geometry and physical characteristics of emitting areas of RBS 1223 (see below). In particular, the obtained parameters of the fits at different phases (Table 2) support a model containing hot areas around the magnetic poles, because the energies of line centers and the blackbody temperatures are higher at the peaks.

In view of this result, we implemented into the X-ray spectral fitting package XSPEC a number of new highly magnetized INS surface/atmosphere models developed in Paper I. They are based on various local models and compute rotational phase-dependent integral emergent spectra of INS, using analytical approximations. The basic model includes temperature/magnetic field distributions over INS surface, viewing geometry, and gravitational redshift. Three local radiating surface models are also considered, namely, a naked condensed iron surface and partially ionized hydrogen model atmospheres, semi-infinite or finite, on top of the condensed surface. Here we reproduce the essential part of the basic model (for details and further references, see Paper I).

To compute an integral spectrum, the model uses an analytical expression for the local spectra: a diluted blackbody spectrum for both semi-infinite and thin models of a magnetized atmosphere with one absorption feature

$I_{\mathrm{E}}(\alpha)=D B_{\mathrm{E}}(T) \phi(\alpha) \exp (-\tau)$,

where $\alpha$ is the angle between radiation propagation direction and the surface normal, and $\phi(\alpha)$ represents the considered angular distributions of the specific intensities for different cases of atmosphere models. We represented $\phi(\alpha)$ by the following three models:

$\phi(\alpha)= \begin{cases}1, & \mathrm{a}, \\ 0.4215+0.86775 \cos \alpha, & \mathrm{b}, \\ \frac{1-0.2 \cos ^{2} \alpha_{\mathrm{c}}}{1-\cos ^{2} \alpha_{\mathrm{c}}} \text { if }\left\{\begin{array}{l}\cos \alpha \geq \cos \alpha_{\mathrm{c}} \\ \text { and } E_{\mathrm{c}, \mathrm{i}}<E<4 E_{\mathrm{C}}, \\ 0.2, \text { if } \cos \alpha<\cos \alpha_{\mathrm{c}} \text { and } E_{\mathrm{c}, \mathrm{i}}<E<4 E_{\mathrm{C}}, \\ 1 \text { for other energies. }\end{array}\right\} \mathrm{c} .\end{cases}$

Here, the cases a, b, and c correspond to the models of isotropic emission, electron scattering directivity pattern, and a finite 

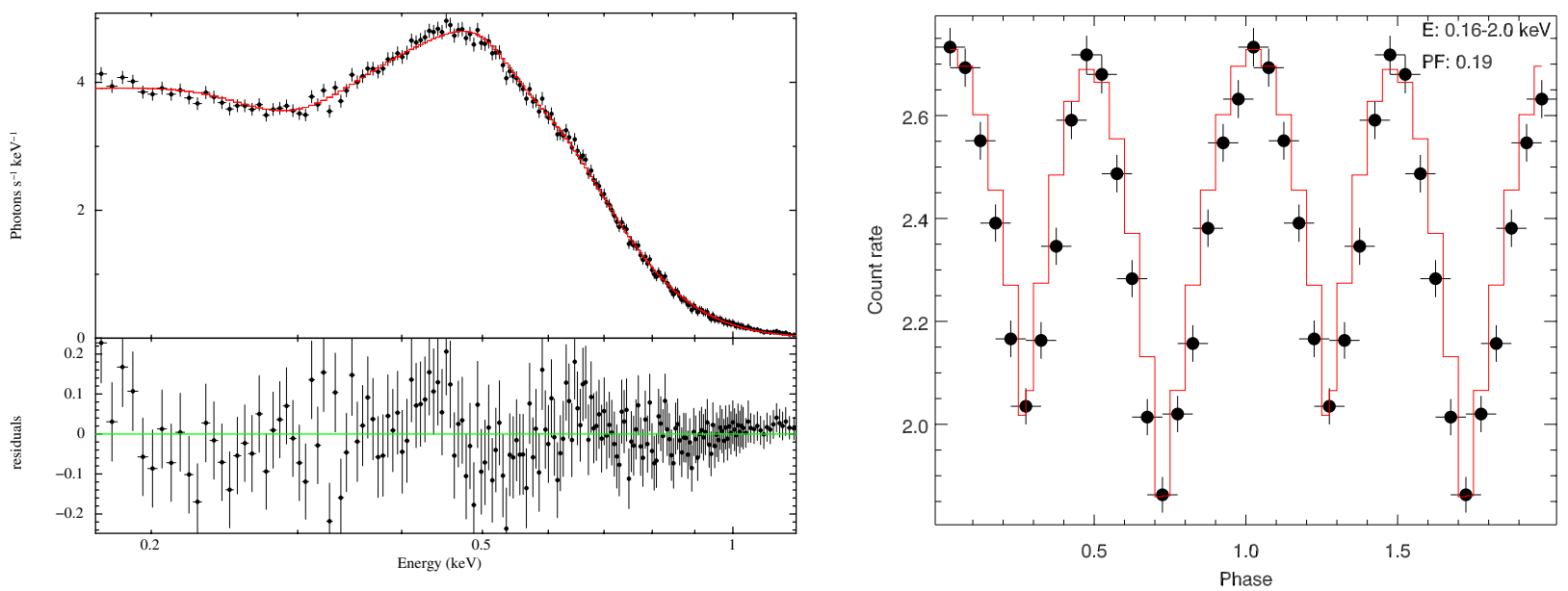

Fig. 3. Phase-averaged X-ray spectrum (left panel) and phase-folded light curve (right panel) in the broad energy band of $0.16-2.0 \mathrm{keV}$ of RBS 1223 combined from 12 pointed XMM-Newton EPIC pn observations.
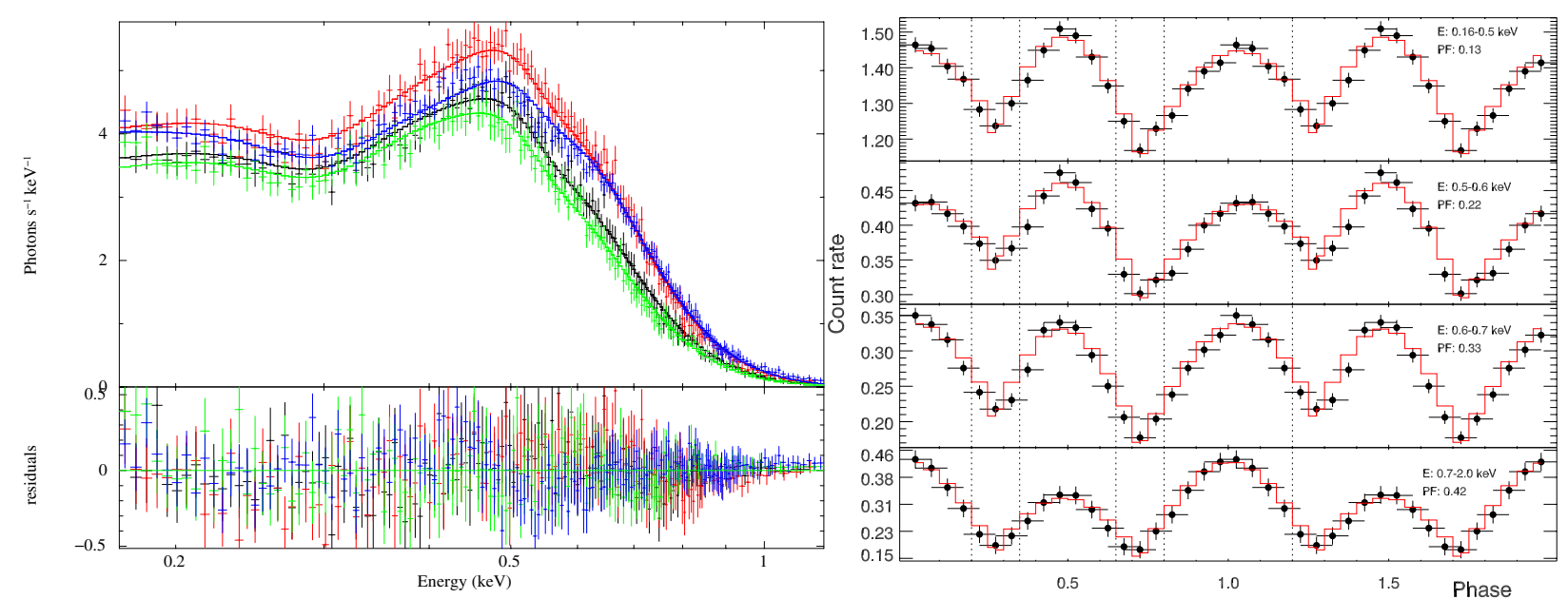

Fig. 4. X-ray spectra including primary and secondary peaks, first and second minima, and phase-folded light curves in different energy bands of RBS 1223 combined from 12 pointed XMM-Newton EPIC pn observations. Fitted absorbed blackbody with Gaussian absorption-line models (phabs*bbodyrad*gabs XSPEC) to the spectra and two sinusoids to the phase-folded light curves are also shown (for details, see text and Table 2). Dotted vertical lines indicate the phase intervals used for the extraction and fitting of spectra shown in the left panel.

hydrogen atmosphere layer above a condensed iron surface, respectively; and the parameter $\cos \alpha_{\mathrm{c}}=\left[\frac{1}{3}\left(E_{\mathrm{C}_{1}} / E_{\mathrm{C}}-1\right)\right]^{2 / 3}$ is the angular distribution in the case of a thin atmosphere for the energy range $E_{\mathrm{ci}}<E<4 E_{\mathrm{C}}$. In addition, $E_{\mathrm{c}, \mathrm{i}}=\hbar \mathrm{ZeB} / \mathrm{m}_{\mathrm{i}} c$ and $E_{\mathrm{c}, \mathrm{e}}=\hbar e B / m_{\mathrm{e}} c$ are the ion and electron cyclotron energies, $E_{\mathrm{p}, \mathrm{e}}=\hbar \sqrt{4 \pi e^{2} n_{\mathrm{e}} / m_{\mathrm{e}}}$ is the electron plasma energy, $Z$ and $m_{\mathrm{i}}$ are the ion charge number and ion mass, $n_{\mathrm{e}}$ is the electron number density, $B$ the magnetic field strength,

$E_{\mathrm{C}}=E_{\mathrm{c}, \mathrm{i}}+E_{\mathrm{p}, \mathrm{e}}^{2} / E_{\mathrm{c}, \mathrm{e}}$,

and

$E_{\mathrm{C}_{1}}=E_{\mathrm{C}}\left(1+3(1-\cos \alpha)^{3 / 2}\right)$.

The optical depths $\tau=\tau^{0} \exp \left(-\frac{\left(E-E_{\text {line }}\right)^{2}}{2 \sigma_{\text {line }}}\right)$ and the widths $\sigma_{\text {line }}$ of the absorption features are considered identical for all local spectra, but the center of the line depends on the local magnetic field strength. In the case of a semi-infinite atmosphere, this absorption line represents the blend of the proton cyclotron line and nearby $b-b$ atomic transitions in neutral hydrogen. The line center corresponds to the proton cyclotron energy $E_{\mathrm{c}, \mathrm{H}}$. In the case of a thin atmosphere, the absorption feature was represented by half a Gaussian line, which means that $\tau^{0}=0$ at $E<E_{\text {line. }}$. This half of the Gaussian line represents a complex absorption feature, which includes a broad absorption feature from the emitting condensed iron surface transmitted through a thin hydrogen atmosphere and the proton cyclotron line plus b-b atomic hydrogen transitions (see details in Paper I). The center of this line corresponds to the ion cyclotron $E_{\mathrm{c}, \mathrm{i}}$ for completely ionized iron. In both cases, the absorption details are better represented by sums of two or even three Gaussian lines. However, we chose to use only one (or half of) a Gaussian line to reduce the number of fitting parameters. The local spectra of the condensed iron surface is approximated by simple step functions (see Paper I). The dilution factor $D$ cannot be found from the fitting independently, and we take $D=1$ for all local spectra.

The blackbody temperature and the magnetic field distributions in the two emitting areas around the magnetic poles were presented analytically by Pérez-Azorín et al. (2006a)

$T^{4}=T_{\mathrm{p} 1,2}^{4} \frac{\cos ^{2} \theta}{\cos ^{2} \theta+a_{1,2} \sin ^{2} \theta}+T_{\min }^{4}$ 
and

$B=B_{\mathrm{p} 1,2} \sqrt{\cos ^{2} \theta+a_{1,2} \sin ^{2} \theta}$,

where $T_{\mathrm{p} 1,2}$ and $B_{\mathrm{p} 1,2}$ are the temperatures and magnetic field strengths at the poles and $a_{1,2}$ their distribution parameters and $T_{\min }$ is the minimum temperature reached on the surface of the star (we chose $\approx 0.3 T_{\mathrm{p}}$, as in Pérez-Azorín et al. 2006a), were $\theta$ is the magnetic colatitude.

The parameters $a_{1,2}$ are approximately equal to the squared ratio of the magnetic field strength at the equator to the field strength at the pole, $a_{1,2} \approx\left(B_{\mathrm{eq}} / B_{\mathrm{p} 1,2}\right)^{2}$. Using these parameters, we can describe various temperature distributions, from strongly peaked $(a \gg 1)$ to the classical dipolar $(a=1 / 4)$ and homogeneous $(a=0)$ ones.

Finally, the total observed flux at distance $d$ from the INS is summed over the visible surface. A local spectrum at photon energy $E$ from the unit surface is computed taking into account viewing geometry, gravitational redshift, and light bending effects (e.g., Poutanen \& Gierliński 2003)

$$
f_{\mathrm{E}} \mathrm{d} \varphi \mathrm{d} \gamma=C_{\text {norm }}(1+z)^{-3} I_{\mathrm{E}^{\prime}}(\alpha) \cos \alpha \sin \gamma \mathrm{d} \varphi \mathrm{d} \gamma,
$$

where $C_{\text {norm }}=R^{2} / d^{2}$ is a normalization constant and $\gamma$ is the angle between the radius vector at a given point and the rotation axis. The observed and emitted photon energies are related as $E=E^{\prime}(1+z)$, where $z$ is the gravitational redshift. The angle $\alpha$ between the emitted photon and normal to the surface in the local reference frame depends on $z$, the local surface position (on azimuthal and colatitude angles $\varphi$ and $\gamma$ ), and the angle between rotation axis and line of sight $i$, and is computed using the approximation of Beloborodov (2002). The summation is performed separately for both regions around magnetic poles, because we allow the poles not to be precisely antipodal (one of them can be shifted by angle $\kappa$ relative to the symmetric antipodal position). Therefore, the poles may have different angles between the rotation and magnetic axes $\theta_{\mathrm{B} 1,2}$.

As shown in Paper I, a model with a thin hydrogen atmosphere above the condensed iron surface with a smooth temperature distribution over the neutron star surface may describe very well the observed physical properties of RBS 1223 .

This basic model has a number of input parameters depending on the inner atmosphere boundary condition of an INS (condensed iron surface or blackbody, temperature and magnetic field distributions over surface, viewing geometry, and gravitational redshift) and angular distribution of the emergent radiation (isotropic or peaked by a thin atmosphere above a condensed surface).

Combined spectra of RBS 1223 in 20 phase bins (Fig. 2) were considered as different data sets during simultaneous fitting with the absorbed abovementioned model. All input parameters were free and linked between those data groups.

Given the large number of the free parameters in the model, we performed a preliminary analysis to roughly estimate (or constrain) some of them. From the observed double-humped light curve shape in different energy bands (see Fig. 4), it has been already clear that two emitting areas have different spectral and geometrical characteristics (e.g., the relatively cooler one has a larger size). Moreover, from the peak-to-peak separation in this double-humped light curve we locate the cooler one at an offset angle of $\kappa$ with respect to the magnetic axis and azimuth (Schwope et al. 2005).

We first performed formal spectral fitting with the simplest model, i.e. absorbed blackbody with a multiplicative Gaussian absorption-line including phases of maxima (see Table 2) in the light curve (Figs. 3 and 4). At these phases, it is clear that X-ray emission is mostly dominated by emitting areas at the magnetic poles, where temperatures also have maxima (see formula of temperature and magnetic field dependence upon polar angle, Pérez-Azorín et al. 2006a, and Paper I). Secondly, some constraints on magnetic field strengths at the poles may be used on the basis of period and its derivative values assuming magnetic dipole breaking as a main mechanism of the spin down of RBS 1223.

With these two general constraints on temperatures and magnetic field strengths at the poles, we simulated a large number of photon spectra (absorbed blackbody with Gaussian absorptionline and different models considered in the Paper I) folded with the response of XMM-Newton EPIC pn camera, taking into account also the interstellar absorption (for parameters see Table 2, and using a characteristic magnetic field strength of $B \sim 3.4 \times 10^{13}$ Gauss).

We cross-correlated the predicted phase-folded light curves in four spectral ranges $0.16-0.5 \mathrm{keV}, 0.5-0.6 \mathrm{keV}, 0.6-0.7 \mathrm{keV}$, and $0.7-2.0 \mathrm{keV}$ and with free parameters of viewing geometry and gravitational redshift, normalized to the maximum of the brightness, with observed ones (Fig. 4). We infer some constraints on the parameters from their unimodal distributions when the cross-correlation coefficients exceeded 0.9 in the aforementioned four energy bands simultaneously and used them as the initial input value and as lower and upper bounds for fitting purposes. For example, gravitational redshift cannot exceed 0.3 (it is impossible to obtain the observed PF at higher $z$ because of strong light bending) or the antipodal shift angle must be less than $25^{\circ}$ (due to the observed phase separation between the two peaks in the light curve). It is also evident that the sum of the inclination angle of the line of sight and magnetic poles relative to the rotational axis are already constrained by the light curve class (see, Poutanen \& Beloborodov 2006, class III) and have the maximum effect (e.g., provide the maximum PF) when both are equal to $90^{\circ}$. We note that these angles do not have a large influence on the fitting, and the only important issue is a range of values when two maxima are observed.

Having the above-mentioned crude constraints and input values of free parameters, we performed fitting with the models implemented in the XSPEC package of combined spectra of RBS 1223 in 20 phase bins (Fig. 2) simultaneously, i.e. each of these phase-resolved spectrum considered as different data sets with the linked parameters to the others, and the only differences were phase ranges, which were fixed for an individual phase-resolved spectrum.

The fitting was successful, with a C-statistic value of 2937 with 2159 degrees of freedom. These parameters are presented in Table 3.

To assess a degree of uniqueness and estimate the confidence intervals of the determined parameters, we have additionally performed Markov chain Monte Carlo (MCMC) fitting as implemented in XSPEC. In Fig. 5, we presented probability density distributions of some of them. We note that independent initial input values of the parameters of the MCMC approach converged to the same values, in six different chains.

\section{Discussion}

We have found that the combined phase-resolved spectra of RBS 1223 can be simultaneously fitted by emergent radiation of a spectral model of an iron condensed surface with a partially ionized hydrogen atmosphere above. The spectra can also be fitted formally by a blackbody spectrum with a proton-cyclotron 
Table 3. Simultaneous fitting results of the combined, phase resolved X-ray spectra of RBS 1223 with different spectral models.

\begin{tabular}{lcc}
\hline \hline $\begin{array}{l}\text { Fitted } \\
\text { parameter }\end{array}$ & \multicolumn{2}{c}{ Spectral model } \\
& $\begin{array}{c}\text { Iron condensed surface } \\
\text { partially ionized H atmosphere }\end{array}$ & $\begin{array}{c}\text { Blackbody } \\
\text { electron scattering }\end{array}$ \\
\hline$T_{\mathrm{p} 1}[\mathrm{eV}]$ & $105.0_{-4.0}^{+2.0}$ & $109 \pm 4.0$ \\
$T_{\mathrm{p} 2}[\mathrm{eV}]$ & $99.0 \pm 3.0$ & $106 \pm 3.0$ \\
$B_{\mathrm{p} 1} \times 10^{14}[\mathrm{G}]$ & $0.86 \pm 0.02$ & $0.65 \pm 0.03$ \\
$B_{\mathrm{p} 2} \times 10^{14}[\mathrm{G}]$ & $0.86 \pm 0.02$ & $0.58 \pm 0.02$ \\
$a_{\mathrm{p} 1}$ & $0.61 \pm 0.11$ & 0.25 \\
$a_{\mathrm{p} 2}$ & $0.29 \pm 0.05$ & 0.25 \\
$\tau^{0}$ & $2.76_{-0.02}^{+0.10}$ & $1.90 \pm 0.06$ \\
$\sigma[\mathrm{eV}]$ & $225.8_{-1.8}^{+5.6}$ & $168.0 \pm 6.0$ \\
$z$ & $0.15 \pm 0.02$ & $0.17 \pm 0.03$ \\
$\kappa\left[^{\circ}\right]$ & $4.2 \pm 0.6$ & $4.0 \pm 0.4$ \\
$i\left[^{\circ}\right]$ & $48.9 \pm 0.5$ & $45.6 \pm 0.5$ \\
$\theta\left[^{\circ}\right]$ & $90.0 \pm 0.5$ & $90.0 \pm 0.5$ \\
\hline
\end{tabular}

Notes. ${ }^{(*)}$ See Sect. 3 for definition of the model parameters.
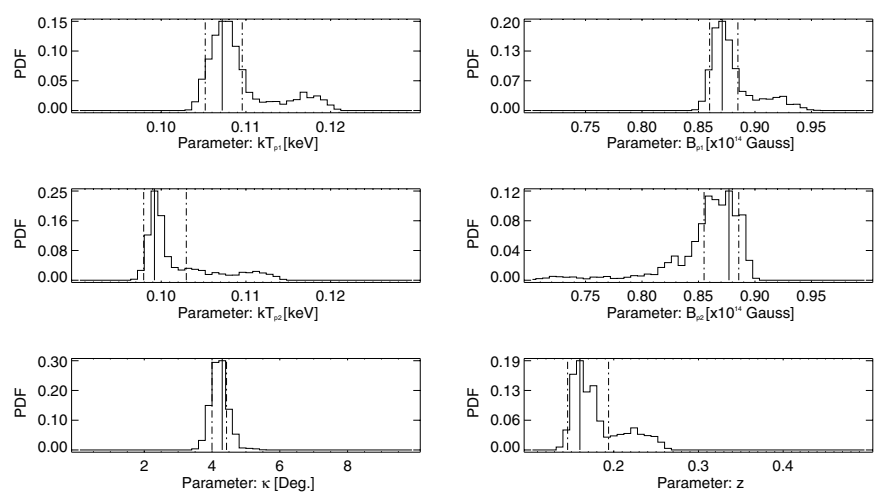

Fig. 5. Probability density distributions of parameters (temperatures, magnetic field strengths at the poles, antipodal shift angle and gravitational redshift) by MCMC fitting with the model of a neutron star with condensed surface and partially ionized hydrogen layer above it. The most probable parameter value is indicated by the solid vertical line. Dashed vertical lines indicate the highest probability interval (68\%, for details see text).

absorption Gaussian line and a peaked (typical of an electronscattering atmosphere) angular distribution of the emergent radiation. In both cases, two emitting areas with slightly different characteristics are required (see Table 3 ).

We note, that the resulting fit parameters are very similar for different spectral models (see Table 3), which is also confirmed by an MCMC approach with different input parameters.

However, we believe that the emission properties of the condensed surface model with a partially ionized, optically thin hydrogen layer above it, including vacuum polarization effects, is more physically motivated. Moreover, semi-infinite atmospheres have rather fan-beamed emergent radiation (see Paper I and references within) and it seems impossible to combine a proton cyclotron line with a pencil-beamed emergent radiation.

We calculated a set of thin highly magnetized partially ionized hydrogen atmospheres above a condensed iron surface with magnetic field strength $B=8 \times 10^{13} \mathrm{G}$, which is close to the value estimated from observations. The observed blackbody temperature of the spectra is reproduced at effective temperatures $T_{\text {eff }} \approx 7 \times 10^{5} \mathrm{~K}$. Examples of the computed emergent

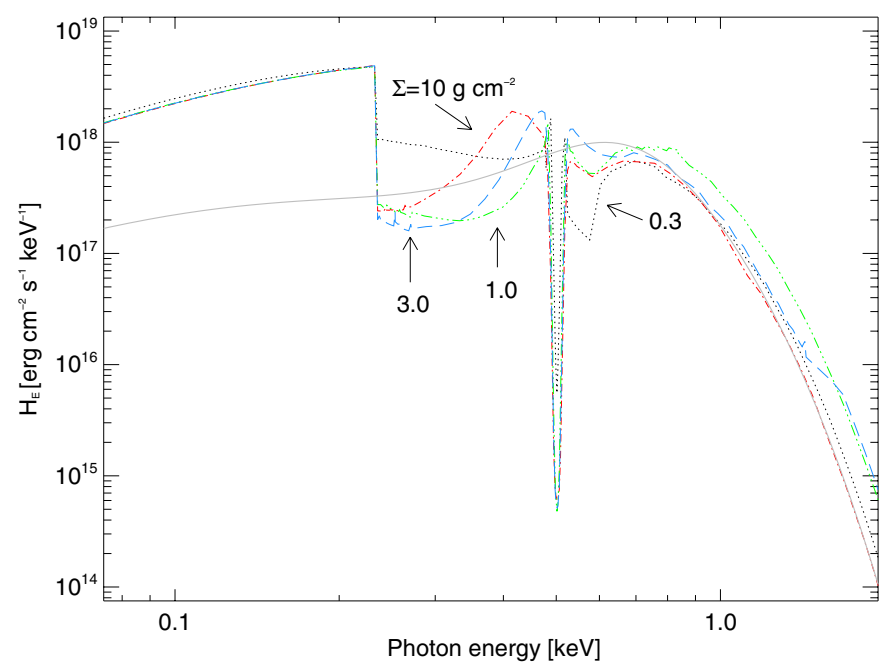

Fig. 6. Emergent spectra of the fitted model of a magnetized atmosphere with a condensed iron surface and a partially ionized hydrogen layer above it (see Table 3). Different lines correspond to different atmospheric surface densities. For comparison purposes, a blackbody spectrum with Gaussian absorption-line is also shown in gray (see text).

spectra with parameters $B=8 \times 10^{13} \mathrm{G}, T_{\text {eff }}=7 \times 10^{5} \mathrm{~K}$, and various atmosphere column densities $\Sigma$ are shown in Fig. 6. The emergent diluted blackbody spectrum with $T=0.1 \mathrm{keV}$ and the absorption Gaussian line parameters, presented in Table 3 $\left(\tau^{0}=2.8, \sigma_{\text {line }}=0.226 \mathrm{keV}, E_{\text {line }}=0.24 \mathrm{keV}\right)$ and $D=0.34$ are also shown. Unfortunately, from these models we cannot evaluate the actual atmosphere thickness (the surface density), but we can obtain the dilution factor $D$, which is important to correct the distance estimation.

Emission spectra based on realistic temperature and magnetic field distributions with strongly magnetized hydrogen atmospheres (or other light elements) are formally still an alternative $^{3}$, but it is unphysical because of the absorption-line added by hand.

A purely proton-cyclotron absorption-line scenario can be excluded owing to the equivalent width of the observed absorption spectral feature in the X-ray spectrum of RBS 1223. Magnetized semi-infinite atmospheres predict too low an equivalent width of the proton cyclotron line in comparison with the observed one.

This result of the fitting (with the condensed surface model with partially ionized, optically thin hydrogen atmosphere above it, including vacuum polarization effects) suggests a true radius of RBS 1223 of $16 \pm 1 \mathrm{~km}$ for a standard neutron star of 1.4 solar mass, considerably larger than the canonical radius of $10 \mathrm{~km}$; it is only marginally compatible with the range from $\approx 10 \mathrm{~km}$ to $\approx 14 \mathrm{~km}$, allowed by modern theoretical equations of state of superdense matter (Haensel et al. 2007; Hebeler et al. 2010, and references therein), and indicates a very stiff equation of state of RBS 1223 (Fig. 7; for similar results, see also Ho et al. 2007; Heinke et al. 2006; Suleimanov \& Poutanen 2006; Suleimanov et al. 2010b).

\footnotetext{
${ }^{3}$ Our attempt to fit the combined, phase-averaged spectrum of RBS 1223 by a partially ionized, strongly magnetized hydrogen or mid$Z$ element plasma model (XSPEC nsmax, Mori \& Ho 2007; Ho et al. 2008 ), as well as two spots or purely condensed iron surface models, failed. Noteworthy, an acceptable fit is obtained by nsmax model with an additional, multiplicative Gaussian absorption-line component (model gabs).
} 


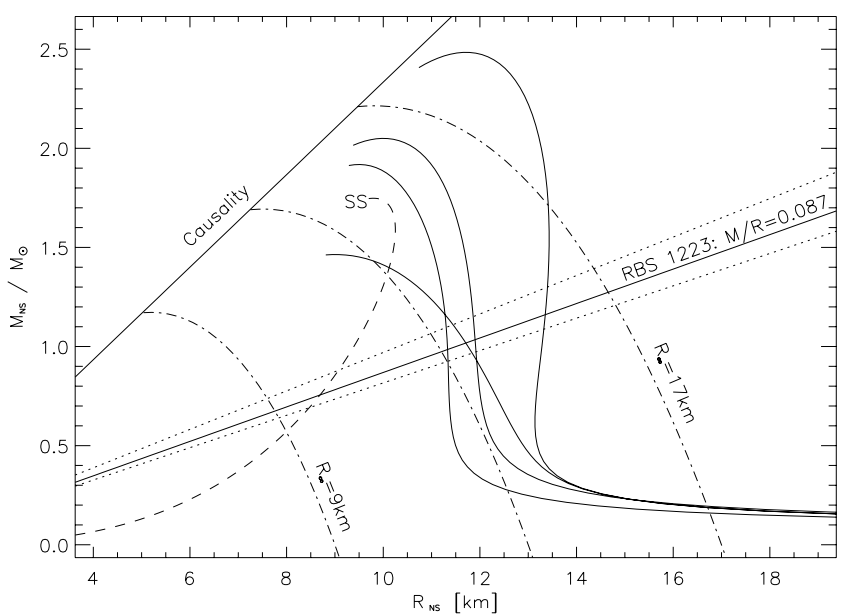

Fig. 7. Mass-radius relations for several equations of state (thin solid curves, Haensel et al. 2007), and a strange star (dashed). Thin dashdoted: curves of constant apparent radius $R_{\infty}=R / \sqrt{1-2 G M / R c^{2}}=9$, 13, and $17 \mathrm{~km}$ (Trümper 2005).

With these estimates of the radius and normalization constant of the fit $\left(R / d \approx 0.0247 \mathrm{~km} \mathrm{pc}^{-1}\right)$, we obtained for the assessment of the distance of RBS $1223 \sqrt{D} 650_{-50}^{+25} \mathrm{pc}=$ $\sqrt{D / 0.34} 380_{-30}^{+15}$ pc (see also Schwope et al. 2005).

\section{Conclusions}

The observed phase-resolved spectra of the INS RBS 1223 have been satisfactorily fitted with two slightly different physical and geometrical characteristics of emitting areas, by the model of a condensed iron surface, with partially ionized, optically thin hydrogen atmosphere above it, including vacuum polarization effects, as an orthogonal rotator. The fit also suggests the absence of a strong toroidal magnetic field component. Moreover, the determined mass-radius ratio $\left(\left(M / M_{\odot}\right) /(R / \mathrm{km})=0.087 \pm 0.004\right)$ suggests a very stiff equation of state of RBS 1223 .

These results on RBS 1223 are promising, since we could find good simultaneous fits to the rotational phase-resolved spectra of RBS 1223 using analytic approximations to the abovementioned model implementation.

It would certainly be helpful to perform more detailed spectral model computation in the near future and apply this to the phase-resolved spectra of other INSs, in particular,
RX J1856.5-3754 and RX J0720.4-3125, including high resolution spectra observed by XMM-Newton and Chandra possibly additional absorption features (Hambaryan et al. 2009; Potekhin 2010).

Acknowledgements. V.H. and V.S. acknowledge support by the German Deutsche Forschungsgemeinschaft (DFG) through project C7 of SFB/TR 7 "Gravitationswellenastronomie". A.Y.P. acknowledges partial support from the RFBR (Grant 11-02-00253-a) and the Russian Leading Scientific Schools program (Grant NSh-3769.2010.2).

\section{References}

Beloborodov, A. M. 2002, ApJ, 566, L85

den Herder, J. W., Brinkman, A. C., Kahn, S. M., et al. 2001, A\&A, 365, L7

Haberl, F. 2007, Ap\&SS, 308, 181

Haensel, P., Potekhin, A. Y., \& Yakovlev, D. G. 2007, Neutron Stars 1: Equation of State and Structure (New York: Springer), Astrophys. Space Sci. Libr., 326 Hambaryan, V., Neuhäuser, R., Haberl, F., Hohle, M. M., \& Schwope, A. D. 2009, A\&A, 497, L9

Hebeler, K., Lattimer, J. M., Pethick, C. J., \& Schwenk, A. 2010, Phys. Rev. Lett., 105, 161102

Heinke, C. O., Rybicki, G. B., Narayan, R., \& Grindlay, J. E. 2006, ApJ, 644, 1090

Ho, W. C. G., Kaplan, D. L., Chang, P., van Adelsberg, M., \& Potekhin, A. Y. 2007, MNRAS, 375, 821

Ho, W. C. G., Potekhin, A. Y., \& Chabrier, G. 2008, ApJS, 178, 102

Ho, W. C. G., Potekhin, A. Y., Chabrier, G., \& Mori, K. 2009, in BAAS, 41, 308

Kaplan, D. L., \& van Kerkwijk, M. H. 2005, ApJ, 635, L65

Mereghetti, S. 2008, A\&AR, 15, 225

Mori, K., \& Ho, W. C. G. 2007, MNRAS, 377, 905

Pérez-Azorín, J. F., Miralles, J. A., \& Pons, J. A. 2006a, A\&A, 451, 1009

Pérez-Azorín, J. F., Pons, J. A., Miralles, J. A., \& Miniutti, G. 2006b, A\&A, 459, 175

Potekhin, A. Y. 2010, A\&A, 518, A24

Poutanen, J., \& Beloborodov, A. M. 2006, MNRAS, 373, 836

Poutanen, J., \& Gierliński, M. 2003, MNRAS, 343, 1301

Schwope, A. D., Hasinger, G., Schwarz, R., Haberl, F., \& Schmidt, M. 1999, A\&A, 341, L51

Schwope, A. D., Hambaryan, V., Haberl, F., \& Motch, C. 2005, A\&A, 441, 597 Schwope, A. D., Hambaryan, V., Haberl, F., \& Motch, C. 2007, Ap\&SS, 308, 619

Suleimanov, V., \& Poutanen, J. 2006, MNRAS, 369, 2036

Suleimanov, V., Potekhin, A. Y., \& Werner, K. 2009, A\&A, 500, 891

Suleimanov, V., Hambaryan, V., Potekhin, A. Y., et al. 2010a, A\&A, 522, A111

Suleimanov, V., Poutanen, J., Revnivtsev, M., \& Werner, K. 2010b, arXiv eprints

Trümper, J. E. 2005, in The Electromagnetic Spectrum of Neutron Stars, ed. A. Baykal, S. K. Yerli, S. C. Inam, \& S. Grebenev, NATO ASIB Proc., 210, 117

Trümper, J. E., Burwitz, V., Haberl, F., \& Zavlin, V. E. 2004, Nucl. Phys. B Proc. Suppl., 132, 560

Turolla, R. 2009, in Neutron Stars and Pulsars, ed. W. Becker, Astrophys. Space Sci. Libr., 357, 141

van Adelsberg, M., \& Lai, D. 2006, MNRAS, 373, 1495 